\title{
CORRECTION
}

\section{Correction to: USP1 inhibition destabilizes KPNA2 and suppresses breast cancer metastasis}

Aihui Ma, Ming Tang, Li Zhang, Boshi Wang, Zhaojuan Yang, Yun Liu, Guiqin Xu, Lin Wu, Tiantian Jing, Xiaoli Xu, Shengli Yang and Yongzhong Liu (D)

(c) The Author(s), under exclusive licence to Springer Nature Limited 2022

Oncogene (2022) 41:1673; https://doi.org/10.1038/s41388-022-02215-y

Correction to: Oncogene https://doi.org/10.1038/s41388-018-0590-8, published online 07 December 2018

Following the publication of this article, the authors noted an error in Fig. 6b. The upper part of the panel was inadvertently inverted. The corrected image is provided below.

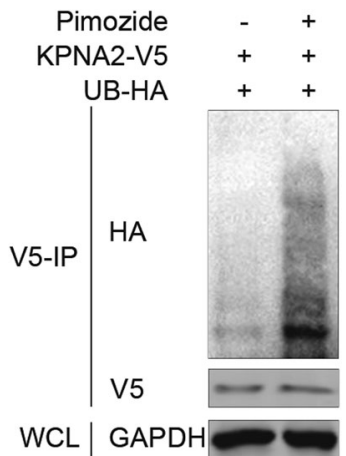

In addition, a sentence on page 2412 has been corrected from 'Consistently, pimozide decreased the levels of ubiquitinated KPNA2 protein and promoted its destabilization (Fig. 6b).' to 'Consistently, pimozide increased the levels of ubiquitinated KPNA2 protein and promoted its destabilization (Fig. 6b).'

The authors confirm these corrections do not alter the conclusions of the paper. 